\section{The effect of different radiological models on diagnostic accuracy and lung cancer screening performance}

\begin{abstract}
High false-positive (FP) scan rates associated with low-dose computed tomography (LDCT) lung cancer screening result in unnecessary follow-up tests and exposure to harm. The definition of a 'positive' scan can impact FP rates and screening performance. We explored the effect of Lung Imaging Reporting and Data System (Lung-RADS) criteria, PanCan Nodule Malignancy Probability Model and varying nodule size thresholds ( $\geq 4 \mathrm{~mm}$, $\geq 6 \mathrm{~mm}, \geq 8 \mathrm{~mm}$ ) on diagnostic accuracy and screening performance compared with original trial definitions (National Lung Screening Trial (NLST) criteria) in a secondary analysis of a lung cancer screening cohort. We found LungRADS criteria and the PanCan Nodule Malignancy Probability Model could substantially improve screening performance and reduce FP scan rates compared with NLST definitions of positivity but that this needs to be balanced against possible risk of falsenegative results.
\end{abstract}

Trial registration number Australian New Zealand Clinical Trials Registry, ACTRN12610000007033.

\section{BACKGROUND}

The landmark National Lung Screening Trial $(\mathrm{NLST})^{1}$ demonstrated lung cancer mortality reduction by low-dose computed tomography (LDCT) screening, paving the way for US Preventative Services Taskforce and Medicare screening recommendations. However, a major limitation of LDCT screening is the high positive scan rate, averaging $24.2 \%$ in NLST (threshold axial diameter $\geq 4 \mathrm{~mm})^{1}$; furthermore, over $95 \%$ of these nodules were benign. ${ }^{1}$ Detected nodules require radiological follow-up creating a burden on healthcare systems and exposing participants to potential harm.

Strategies to improve this situation could include the following: (1) increasing the threshold nodule size, reflecting lower cancer risk in smaller nodules, ${ }^{2}{ }^{3}$ (2) estimating the probability of malignancy using the PanCan multivariable Nodule Malignancy Probability Model (incorporating nodule size, location, attenuation, total count, spiculation, participant age, sex, family history of lung cancer and emphysema, henceforth referred to as the PanCan Model) ${ }^{4}$ and (3) categorisation using the
American College of Radiology Lung Imaging Reporting and Data System (Lung-RADS). ${ }^{5}$ The Lung-RADS classification is based on nodule average diameter, density and growth and also recommends the use of the PanCan Model to guide management of larger nodules (category 4B).

Larger nodule size thresholds are simple to implement but ignore other risk factors. ${ }^{2}{ }^{3}$ The PanCan Model is more complex to administer but appears highly discriminatory ${ }^{4} 67$ and is recommended in British Thoracic Society guidelines ${ }^{8}$ (suggested cut-point for investigation $\geq 10 \%$ risk). The Lung-RADS system is simple to use and improves false-positive rate (1-specificity, FPR) at the cost of lower sensitivity. ${ }^{10}$ Until now, no study has simultaneously compared these competing methods of nodule assessment on false-positive (FP) scan rates and other screening performance metrics.

\section{AIM}

To evaluate the impact of different definitions of positive scan results on screening performance by retrospective application of Lung-RADS, the PanCan Model and various nodule size thresholds to screening cohort data.

\section{METHOD}

Participant eligibility and recruitment have been described elsewhere. ${ }^{11}{ }^{12}$ Two hundred and fifty-six healthy current or former smokers (171 men; 85 women) aged 60-74 years were enrolled in a LDCT screening study; median age 64.5 years; median pack-years of smoking 55 and $47 \%$ current smokers. Participants received baseline scans $\left(\mathrm{T}_{0}\right)$ and up to two annual incidence scans $\left(T_{1}\right.$ and $\left.T_{2}\right)$ using a 64-detector helical CT based on NLST protocols. Criteria for scan positivity, based on NLST, were any nodule $\geq 4 \mathrm{~mm}$ diameter (baseline scan) and any new or growing nodule (incidence scan). ${ }^{11}{ }^{12}$ Indeterminate nodules underwent 2 years radiological follow-up. Health status follow-up continued for 5 years. Data were censored 1 March 2016.

Scan results were reclassified using Lung-RADS (category 3 or 4 considered positive), the PanCan Model (full model with spiculation, $\geq 10 \%$ risk considered positive) and increasing nodule size thresholds (exploratory cut-points set at maximum diameter of $\geq 4 \mathrm{~mm}, \geq 6 \mathrm{~mm}$ or $\geq 8 \mathrm{~mm}$ and labelled $\mathrm{d} 4, \mathrm{~d} 6$ and $\mathrm{d} 8$, respectively). Performance metrics were calculated at the participant level using the scan result and NLST Lung Cancer Status definition (cancer present; cancer absent $\left.{ }^{13}\right)$. Scans were excluded if cancer status could not be determined (eg, participant lost to follow-up). Model discrimination, assessed using the area under the receiver operating characteristic curve (AUC), and calibration assessed by visual plot and Hosmer-Lemeshow test, were calculated at the nodule level.

95\% CIs were calculated for sensitivity, specificity, positive predictive value and negative predictive value (Clopper-Pearson exact method ${ }^{14}$ ) and FP rate reduction (1000 bootstrapped samples). AUCs were estimated and compared non-parametrically using the method of Obuchowski which accounts for clustering of nodules within individuals and correlation between AUC comparisons. ${ }^{15}$ Statistical analysis was performed using R V.3.2.4.

\section{RESULTS}

\section{Baseline scans}

Two hundred and fifty-six participants received $T_{0}$ scans (table 1); 136 participants had 301 nodules (median diameter $5.4 \mathrm{~mm}$, range $4.0-34.0 \mathrm{~mm})$. One hundred and twenty-eight (50.0\%) scans were deemed positive (127 scans without historical images demonstrating nodule stability plus one scan with suspicious non-nodule findings). Lung cancer was diagnosed in five $(2.0 \%)$ participants. One individual with missing lung cancer status was excluded. PanCan, Lung-RADS, d4, $\mathrm{d} 6$ and $\mathrm{d} 8$ correctly identified all participants with lung cancer. PanCan and $\mathrm{d} 8$ produced the greatest reduction in FP scans $(89.4 \%$ reduction, $95 \%$ CI 83.6 to 95.2 and $78.0 \%$ reduction, 95\% CI 69.4 to 86.6 , respectively), improving specificity and positive predictive value without reducing sensitivity or negative predictive value (table 2).

\section{Incidence scans}

One hundred and fifty-six of 472 (33.1\%) incidence scans $\left(239 \mathrm{~T}_{1} ; 233 \mathrm{~T}_{2}\right)$ were positive according to our original criteria (new nodule of any size or growth detected) (table 1). Lung cancer was diagnosed in three participants $(1.3 \%)$ at $\mathrm{T}_{1}$ and four $(1.7 \%)$ at $\mathrm{T}_{2}$. Six individuals without lung cancer status were excluded. Increasing nodule size threshold for a positive scan result reduced FPR but also reduced sensitivity. Lung-RADS and $\mathrm{d} 4$ had similar performance characteristics (table 2).

\section{Downstream effects}

At baseline, the PanCan Model would have avoided 110/127 (86.6\%) interval CT scans and 3/5 positron emission tomography (PET) scans in participants without cancer; Lung-RADS would have 
avoided 75 interval CT scans and no PET scans. At the incidence rounds, The Queensland Lung Cancer Screening Study generated 229 interval CT scans from FP nodules and Lung-RADS would have reduced this number to 44. Across three rounds of screening, Lung-RADS would have avoided 260/356 (73.0\%) interval CT scans, 2/7 (28.6\%) PET scans and 2/3 $(66.7 \%)$ bronchoscopies in participants without cancer. However, Lung-RADS would have misclassified one participant with lung cancer present at the $\mathrm{T}_{2}$ screening round (tables 1 and 3 ).

\section{Model performance}

Ten baseline nodules in eight participants were diagnosed as lung cancer during follow-up (median 29.9 months, range 2.0-69.7). PanCan Model discrimination was very good in the 301 baseline nodules (AUC $0.90 ; 95 \%$ CI 0.75 to 1 ) but not statistically different from Lung-RADS (AUC 0.84 ; $95 \%$ CI 0.69 to 0.98 ; difference in $\mathrm{AUC}=0.06,95 \% \mathrm{CI}-0.04$ to 0.16 , p value $=0.25)$. Visual plot and Hosmer-Lemeshow goodness-of-fit test $\left(\chi^{2}=8.8, \mathrm{df}=8, \mathrm{p}=0.36\right)$ did not indicate significant miscalibration.

\section{DISCUSSION}

As lung cancer screening gains traction internationally, attention focuses on minimising harm and controlling costs by reducing FP scan rates. In this exploratory comparative study, stricter definitions of positivity decreased FP results, improved performance metrics and reduced downstream tests at the risk of increasing falsenegative scans at the incidence round.

Table 1 Lung cancer screening scan results: comparison of Queensland Lung Cancer Screening Study original definitions, Lung-RADS criteria, PanCan Model and differing nodule size thresholds

\begin{tabular}{|c|c|c|c|c|c|c|}
\hline \multirow{2}{*}{$\begin{array}{l}\text { Baseline round } \\
\mathrm{d} 4\end{array}$} & \multicolumn{3}{|c|}{ Positive* } & \multicolumn{3}{|c|}{ Negative } \\
\hline & & $128(50.0)$ & & & & $128(50.0)$ \\
\hline d6 & & $64(25.0)$ & \multicolumn{2}{|c|}{$\begin{array}{l}\text { CT scans } n=256 \\
(100.0)\end{array}$} & \multicolumn{2}{|l|}{$192(75.0)$} \\
\hline d8 & & $32(12.5)$ & & & $224(87.5)$ & \\
\hline Lung-RADS & & $56(21.9)$ & & & $200(78.1)$ & \\
\hline \multirow[t]{2}{*}{ PanCan Model } & \multicolumn{3}{|c|}{$18(7.0)$} & \multicolumn{3}{|c|}{$238(93.0)$} \\
\hline & TP & FP & NA & TN & FN & NA \\
\hline $\mathrm{d} 4$ & $5(2.0)$ & $123(48.0)$ & $0(0.0)$ & $127(49.6)$ & $0(0.0)$ & $1(0.4)$ \\
\hline d6 & $5(2.0)$ & $59(23.0)$ & $0(0.0)$ & $191(74.6)$ & $0(0.0)$ & $1(0.4)$ \\
\hline d8 & $5(2.0)$ & $27(10.5)$ & $0(0.0)$ & $223(87.1)$ & $0(0.0)$ & $1(0.4)$ \\
\hline Lung-RADS & $5(2.0)$ & $51(19.9)$ & $0(0.0)$ & $199(77.7)$ & $0(0.0)$ & $1(0.4)$ \\
\hline PanCan Model & $5(2.0)$ & $13(5.1)$ & $0(0.0)$ & $237(92.6)$ & $0(0.0)$ & $1(0.4)$ \\
\hline \multicolumn{2}{|l|}{ Incidence rounds } & \multicolumn{3}{|l|}{ Positive } & \multicolumn{2}{|l|}{ Negative } \\
\hline \multicolumn{2}{|l|}{ d-All } & \multicolumn{3}{|l|}{$156(33.1)$} & \multicolumn{2}{|l|}{$316(66.9)$} \\
\hline \multicolumn{2}{|l|}{$\mathrm{d} 4$} & $44(9.3)$ & \multicolumn{2}{|c|}{$\begin{array}{l}\text { CT scans } n=472 \\
(100.0)\end{array}$} & \multicolumn{2}{|l|}{$428(90.7)$} \\
\hline \multicolumn{2}{|l|}{ d6 } & $26(5.5)$ & & & \multicolumn{2}{|l|}{$446(94.5)$} \\
\hline \multicolumn{2}{|l|}{$\mathrm{d} 8$} & $17(3.6)$ & & & \multicolumn{2}{|l|}{455 (96.4) } \\
\hline \multirow[t]{2}{*}{ Lung-RADS } & & $46(9.7)$ & & & \multicolumn{2}{|l|}{$426(90.3)$} \\
\hline & TP & $\mathrm{FP}$ & NA & TN & $\mathrm{FN}$ & NA \\
\hline d-All & $7(1.5)$ & 149 (31.6) & $0(0.0)$ & $310(65.7)$ & $0(0.0)$ & $6(1.3)$ \\
\hline d4 & $7(1.5)$ & $37(7.8)$ & $0(0.0)$ & $422(89.4)$ & $0(0.0)$ & $6(1.3)$ \\
\hline d6 & $5(1.1)$ & $21(4.4)$ & $0(0.0)$ & $438(92.8)$ & $2(0.4)$ & $6(1.3)$ \\
\hline d8 & $5(1.1)$ & $12(2.5)$ & $0(0.0)$ & 447 (94.7) & $2(0.4)$ & $6(1.3)$ \\
\hline Lung-RADS & $6(1.3)$ & $39(8.3)$ & $1(0.2)$ & $420(89.0)$ & $1(0.2)$ & $5(1.1)$ \\
\hline $\begin{array}{l}\text { Baseline (TO) and in } \\
\text { Data presented as } \\
\text { Criteria for positive } \\
\text { Imaging Reporting } \\
\text { risk model threshol } \\
\text { Criteria for positive } \\
\text { threshold; d8 } \geq 8 \mathrm{~m} \\
{ }^{*} \text { One baseline scan } \\
\mathrm{FN} \text {, false negative; }\end{array}$ & $\begin{array}{l}\text { nce (T1 a } \\
t \text { (\%). } \\
\text { eline scan } \\
\text { Data Syst } \\
10 \% \text {. } \\
\text { dence sca } \\
\text { areshold a } \\
\text { s positive } \\
\text { alse posit }\end{array}$ & $\begin{array}{l}\text { T2) screening } \\
\geq 4 \mathrm{~mm} \text { thres } \\
\text { categories 3-4 } \\
\text {-All new or gr } \\
\text { Lung-RADS ca } \\
\text { to non-nodul } \\
\text { NA, not asses }\end{array}$ & $\begin{array}{l}\text { ds. } \\
\text {; d6 } \geq 6 \mathrm{~m} \\
\text { d PanCan } \\
\text { g nodule( } \\
\text { ries 3-4. } \\
\text { spicious fir } \\
\text { TN, true r }\end{array}$ & $\begin{array}{l}\text { threshold; d8 } \\
\text { del-Pan-Canad } \\
\text { f any size; d4 } \\
\text { ggs. } \\
\text { ative; TP, true }\end{array}$ & $\begin{array}{l}\mathrm{nm} \text { threshol } \\
\text { multivariabl } \\
\mathrm{mm} \text { thresho } \\
\text { itive. }\end{array}$ & $\begin{array}{l}\text { ng-RADS, Lung } \\
\text { istic-regression } \\
\geq 6 \mathrm{~mm}\end{array}$ \\
\hline
\end{tabular}

At baseline, all methods were highly sensitive. Increasing nodule size thresholds and Lung-RADS improved specificity to 76-89\%, but the PanCan Model had the highest specificity of $94.8 \%$. At incidence scan rounds, stricter size definitions improved specificity and reduced FP scans at the cost of lower sensitivity. FPR was reduced by a similar degree to that seen in a retrospective application of Lung-RADS to NLST data $(52-89 \%$ and $74-92 \%$ reduction at baseline and incidence rounds, respectively, compared with 52\% and $76 \%$, respectively ${ }^{10}$ ).

Since baseline scans have no historical comparison against which to assess growth, they inherently generate more positive scans and downstream tests than incidence scans. ${ }^{1}$ In contrast, incident nodules are more likely to be malignant. ${ }^{16}$ In the absence of a validated multivariable risk model for incidence scans, a more conservative, smaller size threshold compared with the baseline scan, such as recommended by Lung-RADS, seems prudent. However, defining an optimal threshold at prevalence and incidence rounds goes beyond the pure metrics of the test and requires a health economic perspective which may differ between countries and healthcare settings. All measurements in this study were based on maximum axial diameter yet volumetric nodule analysis reportedly gives a more accurate assessment of size and interval growth. The NELSON trial compared diagnostic accuracy between diameterbased management and volume-based management and found similar sensitivity but higher specificity for a volumetric approach $^{17}$ (diameter sensitivity 92.4\% and specificity $90.0 \%$; volumetry sensitivity $90.9 \%$ and specificity $94.9 \%$ ). The diameter measurements were generated from volumetric software results and it is possible this may have underestimated the true difference by avoiding the variability associated with human reader measurements. The true advantage of volumetry, which requires specialised software analysis, against a multivariable risk assessment merits further investigation.

Our study is the first to simultaneously compare these differing methods of scan classification. It has limitations inherent to retrospectivity and relatively small cohort size; however, participants were well characterised and long, near-complete followup made cancer status misclassification unlikely.

In conclusion, we demonstrate lung cancer screening performance and FP scan rate can be improved by varying the definition of a positive scan balanced against 
Table 2 Lung cancer screening scan performance metrics: comparison of Queensland Lung Cancer Screening Study original definitions, Lung-RADS criteria, PanCan Model and differing nodule size thresholds

\begin{tabular}{|c|c|c|c|c|c|}
\hline & Sensitivity (\%) & Specificity (\%) & PPV (\%) & NPV (\%) & FPR reduction (\%) \\
\hline \multicolumn{6}{|l|}{ Baseline scan } \\
\hline $\mathrm{d} 4$ & $100(47.8$ to 100$)$ & 50.8 (44.4 to 57.2 ) & $3.9(1.3$ to 8.9$)$ & $100(97.1$ to 100$)$ & NA \\
\hline $\mathrm{d} 6$ & $100(47.8$ to 100$)$ & 76.4 (70.6 to 81.5$)$ & 7.8 (2.6 to 17.3$)$ & 100 (98.1 to 100$)$ & 52.0 (39.4 to 64.6$)$ \\
\hline $\mathrm{d} 8$ & $100(47.8$ to 100$)$ & 89.2 (84.7 to 92.8 ) & 15.6 (5.3 to 32.8$)$ & $100(98.4$ to 100$)$ & 78.0 (69.4 to 86.6$)$ \\
\hline Lung-RADS & $100(47.8$ to 100$)$ & 79.6 (74.1 to 84.4$)$ & 8.9 (3.0 to 19.6$)$ & $100(98.2$ to 100$)$ & 58.5 (46.5 to 70.5$)$ \\
\hline PanCan Model & $100(47.8$ to 100$)$ & 94.8 (91.3 to 97.2$)$ & 27.8 (9.7 to 53.5$)$ & 100 (98.5 to 100$)$ & 89.4 (83.6 to 95.2 ) \\
\hline \multicolumn{6}{|l|}{ Incidence scan } \\
\hline d-All & 100 (59.0 to 100$)$ & 67.5 (63.0 to 71.8$)$ & 4.5 (1.8 to 9.0$)$ & 100 (98.8 to 100$)$ & NA \\
\hline $\mathrm{d} 4$ & 100 (59.0 to 100$)$ & 91.9 (89.1 to 94.3$)$ & $15.9(6.6$ to 30.1$)$ & 100 (99.1 to 100$)$ & 75.2 (67.0 to 83.4$)$ \\
\hline d6 & 71.4 (29.0 to 96.3$)$ & 95.4 (93.1 to 97.1$)$ & 19.2 (6.6 to 39.4$)$ & 99.5 (98.4 to 99.9$)$ & 85.9 (79.4 to 92.4$)$ \\
\hline d8 & 71.4 (29.0 to 96.3 ) & 97.4 (95.5 to 98.6$)$ & $29.4(10.3$ to 56.0$)$ & 99.6 (98.4 to 99.9$)$ & 91.9 (87.2 to 96.6$)$ \\
\hline Lung-RADS & 85.7 (42.1 to 99.6) & 91.5 (88.6 to 93.9) & 13.3 (5.1 to 26.8 ) & 99.8 (98.7 to 100$)$ & 73.8 (64.9 to 82.7$)$ \\
\hline \multicolumn{6}{|c|}{$\begin{array}{l}\text { Baseline (T0) and incidence (T1 and T2) screening rounds. } \\
\text { Data presented as percentage }(95 \% \mathrm{Cl}) \text {. Lung-RADS, Lung Imaging Reporting and Data System categories } 3-4 ; \text { PanCan Model, Pan-Canadian multivariable logistic-regression risk model } \\
\text { threshold } \geq 10 \% ; \mathrm{d} 4, \mathrm{~d} 6, \mathrm{~d} 8 \text {, size threshold definitions for positive scan-the presence of at least one nodule with maximum axial diameter } \geq 4 \mathrm{~mm}, \geq 6 \mathrm{~mm} \text { and } \geq 8 \mathrm{~mm}, \text { respectively. } \\
\text { At baseline scan, d4 was the definition used in the Queensland Lung Cancer Screening Study; d-All, Queensland Lung Cancer Screening Study definition of positive scan during } \\
\text { incidence round-new or growing nodule of any size. } \\
\text { FPR, false-positive rate; NA, not applicable; NPV, negative predictive value; PPV, positive predictive value. }\end{array}$} \\
\hline
\end{tabular}

Table 3 Downstream tests generated from false-positive (FP) scan results

\begin{tabular}{|c|c|c|c|c|c|c|}
\hline & \multicolumn{6}{|c|}{ Threshold/method } \\
\hline & d-All & Lung-RADS & PanCan & d4 & d6 & d8 \\
\hline Baseline round, FP & 123 & 51 & 13 & - & 59 & 27 \\
\hline Interval CT & 127 & 52 & 17 & - & 63 & 31 \\
\hline CT-guided biopsy & 3 & 3 & 3 & & 3 & 3 \\
\hline PET or PET-CT & 5 & 5 & 2 & - & 5 & 5 \\
\hline Bronchoscopy & 1 & 1 & 1 & - & 1 & 1 \\
\hline Incidence rounds, FP & 149 & 39 & - & 37 & 21 & 12 \\
\hline Interval CT & 229 & 44 & - & 65 & 37 & 23 \\
\hline CT-guided biopsy & 0 & 0 & - & 0 & 0 & 0 \\
\hline PET or PET-CT & 2 & 0 & - & 2 & 2 & 2 \\
\hline Bronchoscopy & 2 & 0 & - & 2 & 2 & 2 \\
\hline
\end{tabular}

Criteria for positive baseline scan: $\mathrm{d} 4 \geq 4 \mathrm{~mm}$ threshold; $\mathrm{d} 6 \geq 6 \mathrm{~mm}$ threshold; $\mathrm{d} 8 \geq 8 \mathrm{~mm}$ threshold; Lung-RADS, Lung Imaging Reporting and Data System categories 3-4 and PanCan Model-Pan-Canadian multivariable logistic-regression risk model threshold $\geq 10 \%$.

Criteria for positive incidence scan: $d$-All new or growing nodule(s) of any size; $d 4 \geq 4 \mathrm{~mm}$ threshold; $d 6 \geq 6 \mathrm{~mm}$

threshold; $\mathrm{d} 8 \geq 8 \mathrm{~mm}$ threshold and Lung-RADS categories 3-4.

possible false-negative results. At baseline, the PanCan Model yielded best results, whereas at incidence scan, $\mathrm{d} 4$ followed by Lung-RADS yielded the best specificity and sensitivity.

\section{Henry M Marshall, Henry Zhao, \\ Rayleen V Bowman, Linda H Passmore, Elizabeth M McCaul, lan A Yang, Kwun M Fong}

University of Queensland Thoracic Research Centre and Department of Thoracic Medicine, The Prince Charles Hospital, Queensland, Australia

Correspondence to Dr Henry M Marshall, University of Queensland Thoracic Research Centre, Department of Thoracic Medicine, The Prince Charles Hospital, Rode Rd, Chermside, QLD 4032, Australia;

henry.marshall@health.qld.gov.au

Acknowledgements The Queensland Lung Cancer Study team based at The University of Queensland Thoracic Research Centre, The Prince Charles Hospital,
Brisbane: Dr John Ayres, BM, FRCR; Dr Jane Crossin, MB BCh, FRCR, FRANZCR; Dr Melanie Lau, MBBS, FRANZCR; Adjunct Professor Richard E Slaughter, MBBS, FRANZCR; Stanley Redmond, Dip App Sci; Deborah Courtney, BN; Dr Steven C Leong, MBBS, FRACP; Dr Morgan Windsor, MBBS, FRACS; Associate Professor Paul V Zimmerman, BSC, MBBS, FRACP, MD. Patients and staff at The Prince Charles Hospital, Brisbane.

Contributors HMM had full access to all of the data in the study and takes responsibility for the integrity of the data and the accuracy of the data analysis. Study concept and design: HMM, KMF, IAY, RVB. Acquisition, analysis or interpretation of data: all authors. Drafting of the manuscript: $\mathrm{HMM}, \mathrm{HZ}$. Critical revision of the manuscript for important intellectual content: KMF, IAY, RVB. Statistical analysis: HMM, HZ. Obtained funding: KMF, IAY, RVB. Study supervision: KMF, IAY, RVB.

Funding National Health and Medical Research Council (Practitioner Fellowship 1019891 (KMF); Career Development Fellowship 1026215 (IAY); Medical PhD
Scholarship 631306 (HMM)); Smart State Project Grant, Queensland Health; National Centre for Asbestos Related Diseases Project Grant and The Prince Charles Hospital Foundation.

\section{Competing interests None declared.}

Ethics approval The Prince Charles Hospital human research ethics committee.

Provenance and peer review Not commissioned; externally peer reviewed.

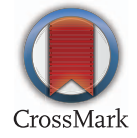

To cite Marshall HM, Zhao H, Bowman R V, et al. Thorax 2017;72:1147-1150.

Received 24 October 2016 Revised 12 February 2017 Accepted 25 February 2017 Published Online First 22 March 2017

Thorax 2017;72:1147-1150. doi:10.1136/thoraxjnl-2016-209624

\section{REFERENCES}

1 Aberle DR, Adams AM, Berg CD, et al., National Lung Screening Trial Research Team. Reduced lung-cancer mortality with low-dose computed tomographic screening. $N$ Engl J Med 2011;365:395-409.

2 Gierada DS, Pinsky P, Nath $\mathrm{H}$, et al. Projected outcomes using different nodule sizes to define a positive CT lung cancer screening examination. J Natl Cancer Inst 2014;106:dju284.

3 Henschke $\mathrm{Cl}$, Yip R, Yankelevitz DF, et al. Definition of a positive test result in computed tomography screening for lung cancer: a cohort study. Ann Intern Med 2013:158:246-52.

4 McWilliams A, Tammemagi MC, Mayo JR, et al. Probability of cancer in pulmonary nodules detected on first screening CT. N Engl J Med 2013;369:910-19.

5 American College of Radiology. Lung CT Screening Reporting and Data System (Lung-RADS). http:// www.acr.org/Quality-Safety/Resources/LungRADS (accessed 21 Oct 2016) 
6 Winkler Wille MM, van Riel SJ, Saghir Z, et al. Predictive accuracy of the PanCan lung cancer risk prediction model -external validation based on $\mathrm{CT}$ from the Danish lung cancer screening trial. Eur Radiol 2015;25:3093-9.

7 Al-Ameri A, Malhotra P, Thygesen $\mathrm{H}$, et al. Risk of malignancy in pulmonary nodules: a validation study of four prediction models. Lung Cancer 2015;89:27-30.

8 Callister MEJ, Baldwin DR, Akram AR, et al. British Thoracic Society guidelines for the investigation and management of pulmonary nodules: accredited by NICE. Thorax 2015;70(Suppl 2):ii1-54.

9 McKee BJ, Regis SM, McKee AB, et al. Performance of $A C R$ Lung-RADS in a clinical CT lung screening program. J Am Coll Radiol 2015;12:273-6.
10 Pinsky PF, Gierada DS, Black W, et al. Performance of Lung-RADS in The National Lung Screening Trial: a retrospective assessment. Ann Intern Med 2015;162:485-91.

11 Marshall HM, Bowman RV, Ayres J, et al. Lung cancer screening feasibility in Australia. Eur Respir J 2015;45:1734-7

12 Marshall HM, Bowman RV, Crossin J, et al. Queensland lung cancer screening study: rationale, design and methods. Intern Med 2013:43:174-82.

13 Church TR, Black WC, Aberle DR, et al., The National Lung Screening Trial Research Team. Results of initial low-dose computed tomographic screening for lung cancer. N Engl J Med 2013;368:1980-91.
14 Clopper CJ, Pearson ES. The use of confidence or fiducial limits illustrated in the case of the binomial. Biometrika 1934;26:404-13.

15 Obuchowski NA. Nonparametric analysis of clustered ROC curve data. Biometrics 1997;53:567-78.

16 Walter JE, Heuvelmans MA, de Jong PA, et al. Occurrence and lung cancer probability of new solid nodules at incidence screening with low-dose $\mathrm{CT}$ : analysis of data from the randomised, controlled NELSON trial. Lancet Oncol 2016;17:907-16

17 Horeweg N, van Rosmalen J, Heuvelmans MA, et al. Lung cancer probability in patients with CT-detected pulmonary nodules: a prespecified analysis of data from the NELSON trial of low-dose CT screening. Lancet Oncol 2014;15:1332-41. 